EPJ Web of Conferences 32, 01012 (2012)

DOI: $10.1051 /$ epjconf/20123201012

(C) Owned by the authors, published by EDP Sciences, 2012

\title{
EC Radiative Transport in the Presence of Anisotropic Distributions of Suprathermal Electrons
}

\author{
F. Albajar ${ }^{1}$, M. Bornatici ${ }^{2}$ and F. Engelmann ${ }^{3}$ \\ ${ }^{1}$ Fusion for Energy ${ }^{*}$, Josep Pla 2, Barcelona, 08019, Spain \\ ${ }^{2}$ Physics Department, University of Pavia, Pavia, 27100, Italy \\ ${ }^{3}$ Max-Planck-Institut für Plasmaphysik, Garching, 85748, Germany
}

\begin{abstract}
The RAYTEC code for electron cyclotron (EC) wave radiative transport modelling of fusion plasmas which earlier had been generalised to include a population of suprathermal electrons to cover EC radiative transport in the presence of an anisotropic distribution of suprathermal electrons in the form of a one-sided loss- or anti-loss-cone in pitch angle, has been modified and, furthermore, now also covers inverted loss-cone and anti-loss-cone distributions. This offers the possibility of dealing with anisotropic distributions symmetric in the electron velocity parallel to the magnetic field, as typical for the case of EC wave heating. Practical issues related to this approach are displayed and the impact of various choices of suprathermal populations on net EC wave power emission from a fusion plasma is evaluated and discussed.
\end{abstract}

\section{Introduction}

The local EC wave power losses can be a competitive contribution to the $1 \mathrm{D}$ electron local power balance for reactor-grade tokamak plasmas with core temperatures of $35 \mathrm{keV}$ or higher as anticipated for steady-state operation in ITER and DEMO [1]. This has motivated work to improve the modelling capabilities for the radial profile of the net EC wave emission in fusion plasmas in view of the calculation of the absorption coefficient [2], the wall reflection properties [3] and polarization scrambling effects [4], the computation efficiency, toroidal plasma geometry with arbitrary crosssection [5], and the presence of a population of suprathermal electrons [6]. The results are implemented in the RAYTEC code [5] for EC wave radiative transport modelling.

In Ref [6] the population of suprathermal electrons was taken to be Maxwellian in energy with part of the distribution having a one-sided loss-cone or anti-loss-cone in pitch angle [7], modelling the cases of suprathermals with an excess of momentum either perpendicular or parallel to the magnetic field. For a well localised anisotropic population of suprathermals the results showed a strong local enhancement of the net emitted EC power density while the total EC radiated power was only slightly increased [6].

\footnotetext{
* The views expressed in this publication are the sole responsibility of the author and do not necessarily reflect the views of Fusion for Energy. Neither Fusion for Energy nor any person acting on behalf of Fusion for Energy is responsible for the use which might be made of the information in this publication.
}

This is an Open Access article distributed under the terms of the Creative Commons Attribution License 2.0, which permits unrestricted use, distribution, and reproduction in any medium, provided the original work is properly cited. 
Both forward and backward (with respect to the magnetic field) loss-cone (and anti-loss-cone) distributions have now been implemented in RAYTEC which offers the possibility of dealing with anisotropic distributions symmetric in the electron velocity parallel to the magnetic field, as typical for the case of EC wave heating.

\section{The model}

The electron momentum distribution is taken in the form,

$$
F(\mathbf{p})=(1-\eta) f\left(p, T_{b}\right)+\eta F_{h}(\mathbf{p})
$$

with

$$
\eta F_{h}(\mathbf{p})=\eta_{h \mathrm{M}} f\left(p, T_{h \mathrm{M}}\right)+\left(\eta-\eta_{h \mathrm{M}}\right) f\left(p, T_{h a}\right) \varphi(\mu=\cos \psi),
$$

where $f\left(p, T_{s}\right)$ is the relativistic Maxwellian $f_{s}\left(p, T_{s}\right)=\mu_{s} /\left(4 \pi K_{2}\left(\mu_{s}\right)\right) e^{-\mu_{s} \gamma}$ where $\mu_{s} \equiv m c^{2} / T_{s}$, $\eta \equiv n_{h} /\left(n_{b}+n_{h}\right)$ denotes the total density of the suprathermals $n_{h}$ normalized to the plasma electron density $n_{t}=n_{b}+n_{h}, n_{b}$ being the bulk electron density; $\eta_{h \mathrm{M}} \equiv n_{h \mathrm{M}} /\left(n_{b}+n_{h}\right)$ is the relative density of suprathermals with an isotropic Maxwellian distribution and temperature $T_{h \mathrm{M}}>T_{b} ; \eta-\eta_{h \mathrm{M}}=n_{h a} n_{t}$ is the (relative) density and $T_{h a}\left(>T_{b}\right.$ ) the temperature of the anisotropic population, which is taken to have a pitch angle distribution $\varphi$ (with normalization $\int_{-1}^{1} d \mu \varphi(\mu)=2$ ) in the form of one of the following distributions (cf. [7]):

$$
\begin{aligned}
& \varphi^{(f)}(\mu)=\frac{1}{1+\mu_{c}}\left[1-\operatorname{erf}\left(\frac{\mu-\mu_{c}}{\sigma \sqrt{2}}\right)\right]\left(=\frac{2}{1+\mu_{c}}\left[1-H\left(\mu-\mu_{c}\right)\right], \text { for } \sigma \rightarrow 0\right), \\
& \varphi^{(b)}(\mu)\left(=\varphi^{(f)}(-\mu)\right)=\frac{1}{1+\mu_{c}}\left[1+\operatorname{erf}\left(\frac{\mu+\mu_{c}}{\sigma \sqrt{2}}\right)\right]\left(=\frac{2}{1+\mu_{c}} H\left(\mu+\mu_{c}\right), \text { for } \sigma \rightarrow 0\right),
\end{aligned}
$$

$\varphi^{(f)}(\mu)$ and $\varphi^{(b)}(\mu)$ representing, respectively, a forward and a backward loss-cone distribution, the last expression within the round brackets referring to the limit of a sharp-edged loss-cone $(H(x)=1$ for $x>0$ and zero otherwise, is the Heaviside step function) as well as the symmetric loss-cone distribution.

$$
\begin{array}{r}
\varphi^{(s)}(\mu)=\frac{1}{2}\left[\varphi^{(f)}(\mu)+\varphi^{(b)}(\mu)\right]=\frac{1}{2\left(1+\mu_{c}\right)}\left[2+\operatorname{erf}\left(\frac{\mu+\mu_{c}}{\sigma \sqrt{2}}\right)-\operatorname{erf}\left(\frac{\mu-\mu_{c}}{\sigma \sqrt{2}}\right)\right] \\
=\frac{1}{1+\mu_{c}}\left[1+H\left(\mu+\mu_{c}\right)-H\left(\mu-\mu_{c}\right)\right], \text { for } \sigma \rightarrow 0,
\end{array}
$$

Fig. 1 shows the distributions of Eqs.(3) and (4).
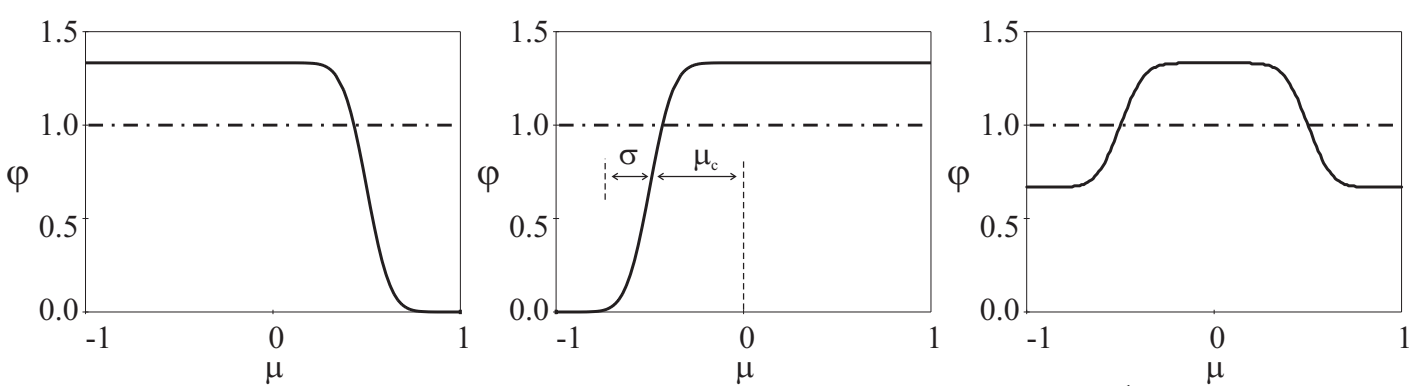

Fig. 1. The pitch angle distribution function $\varphi\left(\mu=\cos \psi\right.$ ) for $\mu_{c}=0.5$ (a cone angle $\cos ^{-1} \mu_{c}=60^{\circ}$ ) and an edge width $\sigma=0.1$ for the forward (left-hand side plot), the backward (middle plot), and the symmetric loss-cone (right-hand side plot). The dotted-dashed line corresponds to the isotropic distribution $\varphi(\mu)=1$ present for $\mu_{c} \rightarrow 1$ and $\sigma \rightarrow 0$. Note that the symmetric loss-cone distribution of Eq.(4) is isotropic, $\varphi^{(s)}(\mu)=1$, also for $\mu_{c}=0$ and $\sigma \rightarrow 0$. 
It is worth noting that the symmetric distribution (4) is an even function of $\mu$ with the result that the corresponding current density parallel to the magnetic field, $j_{\|} \sim \int_{-1}^{1} d \mu \mu \varphi^{(s)}(\mu)$, is zero. The net EC power per unit volume effectively radiated is [8]

$$
\frac{d P(\mathbf{r})}{d V}=\sum_{i=X, O} \int_{\omega_{\min }^{i}}^{\infty} d \omega \int d^{2} \Omega_{\mathrm{s}} \alpha^{(i)}(\mathbf{r}, \omega, \mathbf{s})\left[\left(N_{r}^{(i)}\right)^{2} \frac{\omega^{2}}{8 \pi^{3} c^{2}} T_{\mathrm{rad}}^{(\mathrm{i})}(\mathbf{r}, \omega, \mathbf{s})-I^{(i)}(\mathbf{r}, \omega, \mathbf{s})\right],
$$

where the (total) absorption coefficient $\alpha^{(i)}$ can be approximated [7] by

$$
\begin{gathered}
\alpha^{(i)}=\alpha_{b}^{(i)}+\alpha_{h \mathrm{M}}^{(i)}\left(1+\frac{\eta-\eta_{h \mathrm{M}}}{\eta_{h \mathrm{M}}} A^{(1)}\left(1+R^{(i)}\right)\right), \quad A^{(1)}=\frac{2+\operatorname{erf}\left(x_{+}\right)+\operatorname{erf}\left(x_{-}\right)}{2\left(1+\mu_{c}\right)}, \\
R^{(i)}=\frac{2}{\pi^{1 / 2}} \frac{h^{2}}{\sigma^{2}+h^{2}} \frac{x^{+} \exp \left(-x_{+}^{2}\right)+x^{-} \exp \left(-x_{-}^{2}\right)}{2+\operatorname{erf}\left(x_{+}\right)+\operatorname{erf}\left(x_{-}\right)} \frac{1}{\beta_{0}^{2} \gamma_{0}}\left[\frac{d \ln f(p)}{d \gamma}\right]^{-1}
\end{gathered}
$$

with $x_{ \pm}$being given by Eq.(42) along with Eqs.(41a,b,c) and (21) of [7]. Note that for expression (6) to be valid, $\left|1 \pm \mu_{c}\right| \gtrsim 3 \sigma$ has to hold which excludes taking the (singular) limit $\mu_{c} \rightarrow-1$. Furthermore, with reference to Eq.(5),

$$
T_{\text {rad }}^{(\mathrm{i})}=\frac{T_{b} \alpha_{b}^{(i)}+T_{h} \alpha_{h \mathbf{M}}^{(i)}\left(1+\frac{\eta-\eta_{h \mathbf{M}}}{\eta_{h \mathbf{M}}} A^{(1)}\right)}{\alpha^{(i)}}
$$

is the radiation temperature, $T_{h}\left(=T_{h \mathrm{M}}=T_{h a}\right)$ being the temperature of the suprathermal populations taken to be equal in the the sequel, and $I^{(i)}(\mathbf{r}, \omega, \mathbf{s})$ is the solution of the radiative transfer equation [8]. Note that the $\theta$-integration in (5) $\left(d^{2} \Omega_{s}=2 \pi \sin \theta d \theta\right.$ with $\theta$ the angle between the ray direction $\mathbf{s}$ and the magnetic field) has to be carried out over the whole $(0, \pi)$-range as a consequence of the asymmetry between the $(0, \pi / 2)$-range and the $(\pi / 2, \pi)$-range for an asymmetric electron distribution (see Figures 2) which had not been taken account of in the calculations presented in [6].

For reference, the average perpendicular (to the magnetic field) energy density of the suprathermals is, in the non-relativistic limit and to lowest order in $\sigma^{2}$, i.e., in the limit of a sharpedged loss-cone

$$
E_{\perp}=<\frac{m v_{\perp}^{2}}{2}>=\left(n_{h \mathbf{M}}+n_{h a}\right) T_{h}+\frac{\mu_{c}\left(1-\mu_{c}\right)}{2} n_{h a} T_{h}
$$

independently of the specific form of the erf-cone distribution. In particular one has $E_{\perp}=n_{h \mathbf{M}} T_{h}$ for $\mu_{c}=-1$ as in this case the anisotropic part of the superthermal population degenerates to a beam parallel to the magnetic field. Furthermore, $E_{\perp}=\left(n_{h \mathrm{M}}+n_{h a}\right) T_{h}=n_{h} T_{h}$ for both $\mu_{c}=0$ and $\mu_{c}=1$, as in these limits the anisotropic distribution tends to be, respectively, a half and a full Maxwellian.

\section{Numerical results and discussion}

Prior to evaluating the locally radiated net EC power per unit volume (5), let us examine this quantity per unit solid angle about the ray direction $\mathbf{s}, d^{2} P / d V d \Omega_{\mathbf{s}}$, and more specifically its dependence on the angle $\theta$ between $\mathbf{s}$ and the magnetic field, shown in the left-hand side plot of Fig.2. Here (and in the following) ITER-like plasma parameters are taken with plasma major and minor radius $6.2 \mathrm{~m}$ and $2.0 \mathrm{~m}$, respectively and toroidal magnetic field $5.3 \mathrm{~T}$. The bulk and superthermal electron temperature and density profiles are taken to follow the expressions given in Fig.1 of [6], with $T_{b}(0)=25 \mathrm{keV}, T_{b}(1)=2 \mathrm{keV}, n_{t}(0)=10^{20} \mathrm{~m}^{-3}, n_{t}(1)=5 \times 10^{19} \mathrm{~m}^{-3}$. The effective wall reflection coefficient is taken to be equal to 0.6. The suprathermal populations, for displaying the characteristic features clearly, are taken very high, viz., $\eta=1$; their (Maxwellian) temperature is 
$T_{\mathrm{h}}(0)=75 \mathrm{keV}$. The asymmetry in $\theta$ about $\theta=\pi / 2$ of the EC radiation for both the forward and the backward loss-cone distribution is clearly visible (of course the dependences are specularly symmetric to each other). For a quantitative appreciation of the $\theta$-asymmetry, the relative deviation of $\left(d^{2} P / d V d \Omega_{\mathrm{s}}\right)$ for the forward (f) and backward (b) loss-cone distribution, Eqs.(3)(4), from the isotropic Maxwellian case (Max), is shown on the right-side plot of Fig.2, namely $\Delta(\%)=\left[\left(d^{2} P / d V d \Omega\right)^{(\text {Max })}-\left(d^{2} P / d V d \Omega\right)^{(\mathrm{f}, \mathrm{b})}\right] /\left(d^{2} P / d V d \Omega\right)^{(\text {Max })}$. The $\theta$-asymmetry increases for decreasing $\mu_{\mathrm{c}}$ attaining its minimum (maximum) for $\mu_{c} \rightarrow 1\left(\mu_{c} \rightarrow-1\right)$.
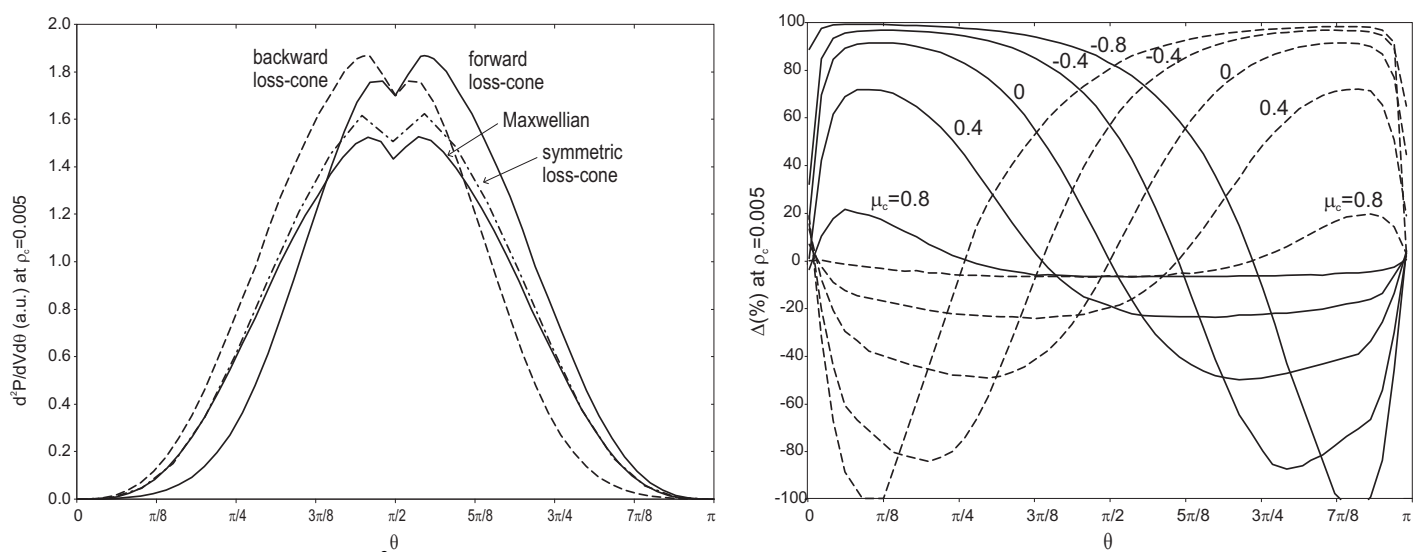

Fig. 2. Left-hand side plot: $d^{2} P / d V d \theta$ vs $\theta$ for the forward, backward and symmetric loss-cone populations for $\eta_{h \mathrm{M}}=0$ and $\mu_{c}=0.4$ and for an isotropic Maxwellian $\left(\eta_{h \mathrm{M}}=\eta=1\right)$. Right-hand side plot: relative (to the Maxwellian) degree $\Delta$ of $\theta$-asymmetry for the forward (full curves) and the backward loss-cone (dashed curves) populations.

In Fig.3, the locally radiated net EC power density $\mathrm{dP} / \mathrm{dV}$ as a function of the loss-cone angle parameter $\mu_{\mathrm{c}}$ is compared for forward, backward and symmetric suprathermal populations on and off axis, again for comparatively high densities $\left(\eta=1 / 10, \eta_{\mathrm{hM}}=(2 / 3) \eta=1 / 15\right)$. The spatial distribution of the suprathermals is Gaussian centered at $\rho_{\mathrm{c}}$ with width $\Delta \rho$ ( $\rho$ denoting the normalised radial coordinate) [6]. These results extend and correct those presented in Ref.[6]. As to be expected, for the symmetric population $d P / d V$ is equal to the value for an isotropic distribution at $\mu_{\mathrm{c}}=0$ and 1 . Note that $\mu_{\mathrm{c}}=0$ separates the loss-cone distributions from anti-loss-cone, i.e. beam-like ones. For the forward and the backward loss-cone the result is equal, for reasons of symmetry. As pointed out in Sect.2, when $\mu_{\mathrm{c}}$ approaches -1 , the model adopted breaks down. Since then the anisotropic population tends to become a beam parallel to the magnetic field, its emissivity vanishes and, for the parameters chosen, its absorptivity is comparatively very small so that in that limit $d P / d V$ is well approximated by the sole contributions of the bulk plasma and the isotropic suprathermal population. In Fig. 3, the results are presented taking account of this consideration.

In Fig.4, the radial dependence of $d P / d V$ is shown for these cases. For the anisotropic populations $\mu_{c}=0.4$ has been chosen. In spite of the quite high density of the anisotropic populations, no significant difference between the symmetric and the asymmetric populations is found. For the isotropic Maxwellian for which the overall perpendicular energy is lower, the local emission is somewhat smaller. 

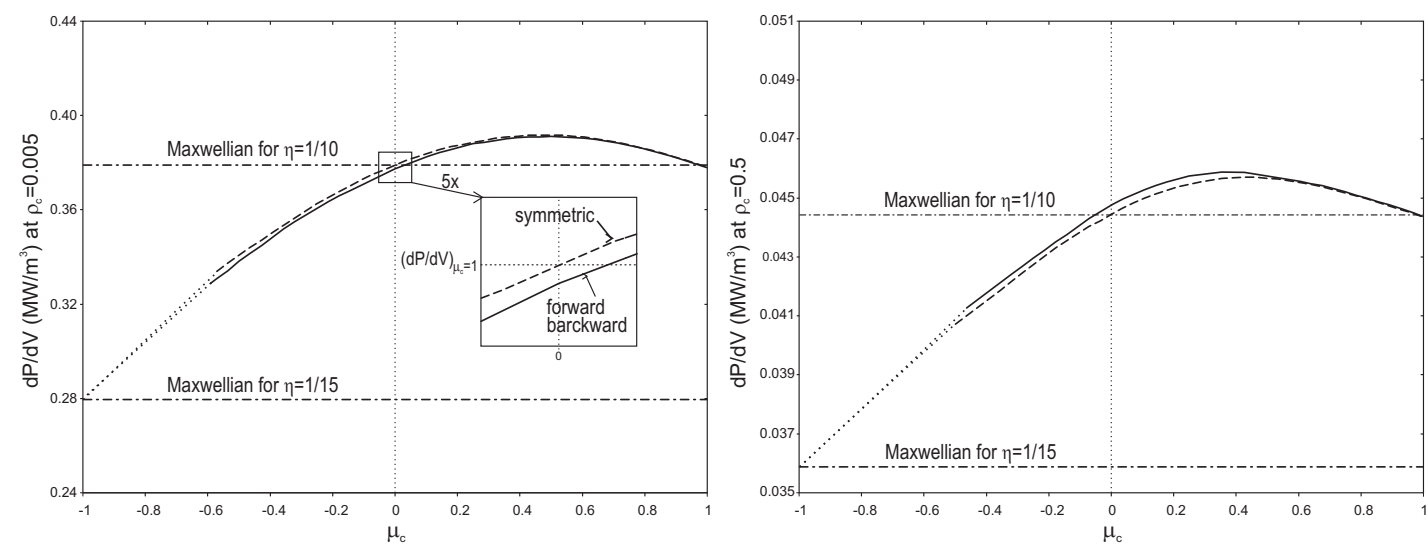

Fig. 3. Locally radiated net EC power density $d P / d V$ for forward and backward (solid), and symmetric (dashed) loss-cone distributions as a function of the loss-cone angle parameter $\mu_{\mathrm{c}}$ for an on-axis $\left(\rho_{\mathrm{c}}=0.005\right)$ and an offaxis $\left(\rho_{\mathrm{c}}=0.5\right)$ population of suprathermals for $\eta=1 / 10, \eta_{h \mathrm{M}}=(2 / 3) \eta=1 / 15, \sigma=0.001$, and, respectively, $T_{\mathrm{h}}(0)=75 \mathrm{keV}$, and $\Delta \rho=0.2$ for the on-axis and $T_{\mathrm{h}}(0.5)=30 \mathrm{keV}$, and $\Delta \rho=0.1$ for the off-axis case. The result for isotropic Maxwellians $\left(\eta_{h \mathrm{M}}=\eta=1 / 10\right.$ and $\left(\eta_{h \mathrm{M}}=(2 / 3) \eta=1 / 15\right)$ is also shown.
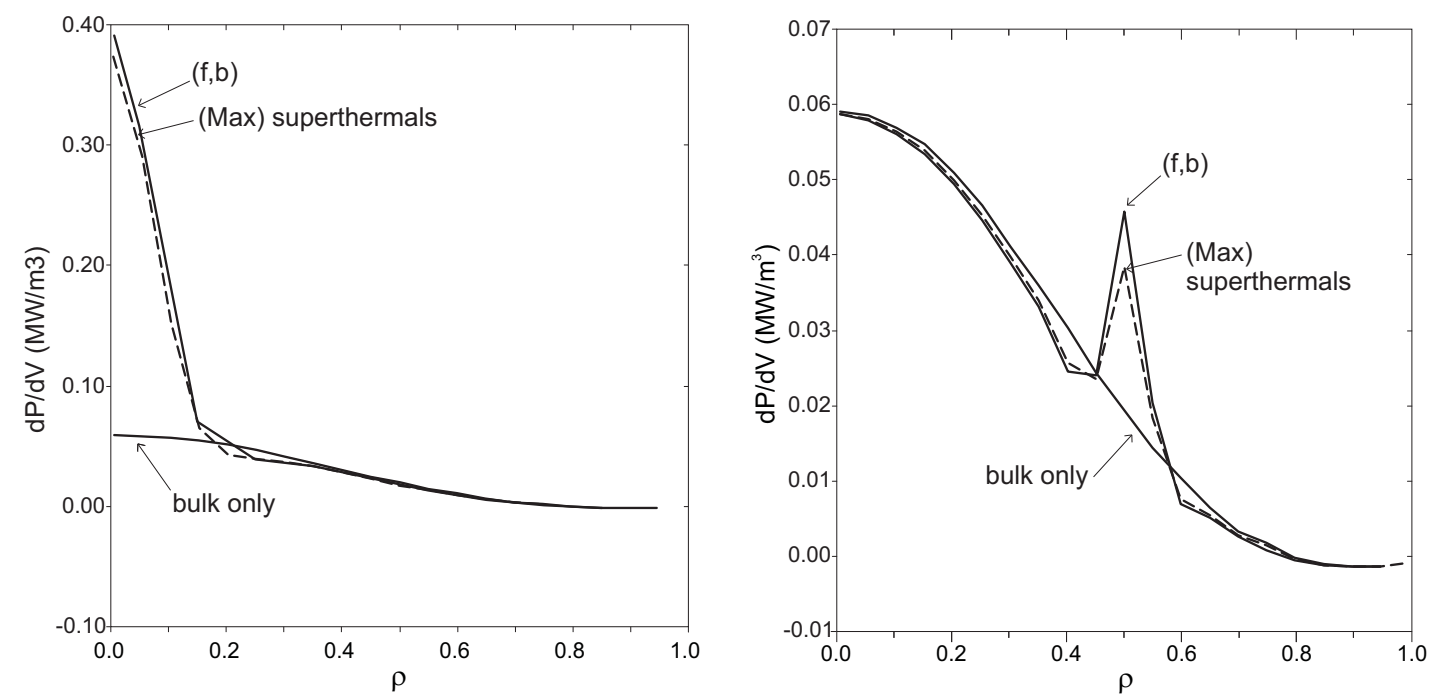

Fig. 4. Radial dependence of $d P / d V$ for on-axis (left-hand side) and off-axis (right-hand side) suprathermal populations with $\mu_{c}=0.4$ as well as for suprathermal isotropic Maxwellian (parameters as in Fig. 3; the isotropic Maxwellian case corresponds to $\eta_{h \mathrm{M}}=\eta=1 / 10$, the bulk only corresponds to the case without suprathermals $\eta=0$ ).

\section{Conclusions}

In addition to the one-sided loss-cone distributions for suprathermal electrons available in the RAYTEC code so far [6], inverted loss-cone distributions have been implemented. This enhancement, in particular, allows treating EC radiation from anisotropic suprathermal electron populations that are symmetric in the electron velocity parallel to the magnetic field as typical for EC wave heating situations. The code was used to display the impact of different assumptions on the anisotropic electron population. Overall, this impact tends to be weak. 


\section{References}

1. F. Albajar et al., Nucl. Fusion 45 (2005) 642-648

2. F. Albajar, M. Bornatici and F. Engelmann, Nucl. Fusion 47 (2007) 1101-1105

3. F. Albajar, M. Bornatici, F. Engelmann and A. B. Kukushkin, Fusion Science and Technology 55 (2009) 76-83

4. F. Albajar, M. Bornatici and F. Engelmann, Nucl. Fusion 45 (2005) L9-L13

5. F. Albajar, M. Bornatici and F. Engelmann, Nucl. Fusion 49 (2009) 115017

6. F. Albajar, M. Bornatici and F. Engelmann, Proceedings of the $16^{\text {th }}$ Joint Workshop on Electron Cyclotron Emission and Electron Cyclotron Resonance Heating (EC-16) (2010, Sanya, China), ed. R. Prater (Singapore: 2011 World Scientific), pp.215-221.

7. P. A. Robinson, Plasma Phys. Control. Fusion 27 (1985) 1037

8. F. Albajar, M. Bornatici and F. Engelmann, Nucl. Fusion 42 (2002) 670-678 